\title{
Overview of the Preliminary Design of the Optical Communication Demonstration and High-Rate Link Facility
}

\author{
J. V. Sandusky, M. Jeganathan, G. Ortiz, A. Biswas, S. Lee, G. Parker, B. Liu. D. Johnson, J. DePew, \\ and J. R. Lesh \\ Optical Communications Group \\ Jet Propulsion Laboratory \\ Califomia Institute of Technology \\ 4800 Oak Grove Drive \\ Pasadena, CA $91109-8099$
}

\begin{abstract}
This paper presents an overview of the preliminary design of both the flight and ground systems of the Optical Communication Demonstration and High-Rate Link Facility which will demonstrate optical communication from the International Space Station to ground after its deployment in October 2002. The overview of the preliminary design of the Flight System proceeds by contrasting it with the design of the laboratory-model unit, emphasizing key changes and the rationale behind the design choices. After presenting the preliminary design of the Ground System, the timetable for the construction and deployment of the flight and ground systems is outlined.
\end{abstract}

Keywords: optical communication, International Space Station, lasercomm

\section{INTRODUCTION}

Motivated by demands for faster, better, cheaper spacecraft, NASA is developing optical communication technology which promises reduced mass, volume, and power consumption compared to radio-frequency technology'. As part of that technology development, the International Space Station (ISS) Engineering Research and Technology Development program (ISSERT) has sponsored the Jet Propulsion Laboratory (JPL) to create the Optical Communication Demonstration and High-Rate Link Facility (OCDHRLF) ${ }^{2}$ which will demonstrate a 2.5 Gbps optical downlink from the ISS to JPL's Optical Communication Telescope Laboratory (OCTL) ${ }^{3}$. This demonstration will gather statistics on the space-to-ground optical communication link and quantify the performance of the OCDHRLF as a system. Following successful demonstration, the high-rate downlink capability will be available as a facility experiment for the downlink of high-rate science data from the ISS.

In this paper presents an overview of the preliminary design of the OCDHRLF. The overview begins with Section 2 which summarizes the link in a design table and the performance of the end-to-end system. Section 3 contrasts the preliminary design of the Flight System in terms of modulation rate, wavelength, output power, instrumentation, and interface to the host platform with the heritage design of the Optical Communication Demonstrator $(O C D)^{4-6}$. Section 4 presents the preliminary design of the Ground System and discusses operations. Finally, Section 5 summarizes the schedule for the development of the OCDHRLF.

Send all correspondence to John Sandusky

email: John.V.Sandusky @jpl.nasa.gov; telephone: 818-354-9388; fax: 818-393-6142 


\begin{tabular}{l|r|r} 
Data Rate & $1.244 \mathrm{Gbps}$ & $2.488 \mathrm{Gbps}$ \\
\hline Transmit Power, average & $23 \mathrm{dBm}$ & $23 \mathrm{dBm}$ \\
Transmit Gain \& Optics Efficiency & $100 \mathrm{~dB}$ & $100 \mathrm{~dB}$ \\
Channel Mis-Pointing Allowance & $-4 \mathrm{~dB}$ & $-4 \mathrm{~dB}$ \\
Space Loss & $-258 \mathrm{~dB}$ & $-258 \mathrm{~dB}$ \\
Atmospheric Transmission Loss & $-2 \mathrm{~dB}$ & $-2 \mathrm{~dB}$ \\
Atmospheric-Induced-Fade Allowance & $-6 \mathrm{~dB}$ & $-6 \mathrm{~dB}$ \\
Receive Gain \& Optics Efficiency & $118 \mathrm{~dB}$ & $118 \mathrm{~dB}$ \\
Received Signal & $-29 \mathrm{dBm}$ & $-29 \mathrm{dBm}$ \\
Required Signal Level at Receiver & $-35.5 \mathrm{dBm}$ & $-31.9 \mathrm{dBm}$ \\
\hline & & \\
Link Margin & $+6.5 \mathrm{~dB}$ & $+2.9 \mathrm{~dB}$
\end{tabular}

Figure 1. This table summarizes, under pessimistic estimations, the gains and losses in the end-to-end optical link at 1.244 and $2.488 \mathrm{Gbps}$ under the worst-cast geometry for a clear day. The gains exceed the losses by approximately $6.5 \mathrm{~dB}$ at $1.244 \mathrm{Gbps}$ and $2.9 \mathrm{~dB}$ at 2.488 Gbps.

\section{END-TO-END SYSTEM}

The end-to-end OCDHRLF design is capable of communicating a pseudorandom bit sequence (PRBS) to the Ground System at a data rate exceeding 1 Gbps and provides a User Input Port by which an externally-supplied, formatted binary data stream can be communicated to the Ground System at similary high data rates, supporting the facility experiment purpose of the OCDHRLF. In addition to these data rate performance goals, the design meets the NASA safety requirement that at the ISS both the intensity of the beacon beam and the intensity of the downlink beam be below the eye-safe limit. The design of the Flight System also includes instrumentation to measure the Flight System's performance in the ISS operating environment and to provide diagnostic capability.

The design of the flight and ground systems were guided by the link table shown in Figure 1. The table is tabulated for an on-off-keyed (OOK) bit stream with a bit-error-rate (BER) of $10^{-6}$, clear weather with light high cirrus clouds, and the worst-case link geometry which occurs when the ISS is $985 \mathrm{~km}$ away from the Ground System. In this link geometry, the ISS is only 20 degrees above the horizon, maximizing the atmospheric transmission loss. The $200 \mathrm{~mW}$ of transmit power is produced by a $1550-\mathrm{nm}$ erbium-doped fiber amplifier arranged in a master-oscillator power-amplifier configuration?. The power from this downlink laser is relayed through an optical system culminating in its collimation by a 10-cm-diameter Cassegrain telescope similar to the telescope used in the $O C D$. The allowance for $4 \mathrm{~dB}$ of pointing loss accounts for the anticipated worst-case residual pointing error associated with misalignments and the finite ability of the fine-steering control loop to compensate for the ISS vibration environment. The design table includes an allowance of $6 \mathrm{~dB}$ for expected intermittent signal reduction due to atmospheric fades. The receiver gain and optics efficiency is based on pessimistic performance estimates of the one-meter-aperture OCTL facility and the receiver sensitivity is asspecified for a commercial-off-the-shelf (COTS) fibercomm-standard receiver. Under these estimates and allowances, more than $6 \mathrm{~dB}$ of margin remains. The end-to-end system utilizes the Synchronous Optical Network (SONET) standard data rates $2.488 \mathrm{Gbps}, 1.244 \mathrm{Gbps}, 622 \mathrm{Mbps}$, and $155 \mathrm{Mbps}$. The link margin increases by approximately $3 \mathrm{~dB}$ with each factor-of-two decrease in data rate; approximately $3 \mathrm{~dB}$ of link margin remains at the highest data rate of 2.488 Gbps. 


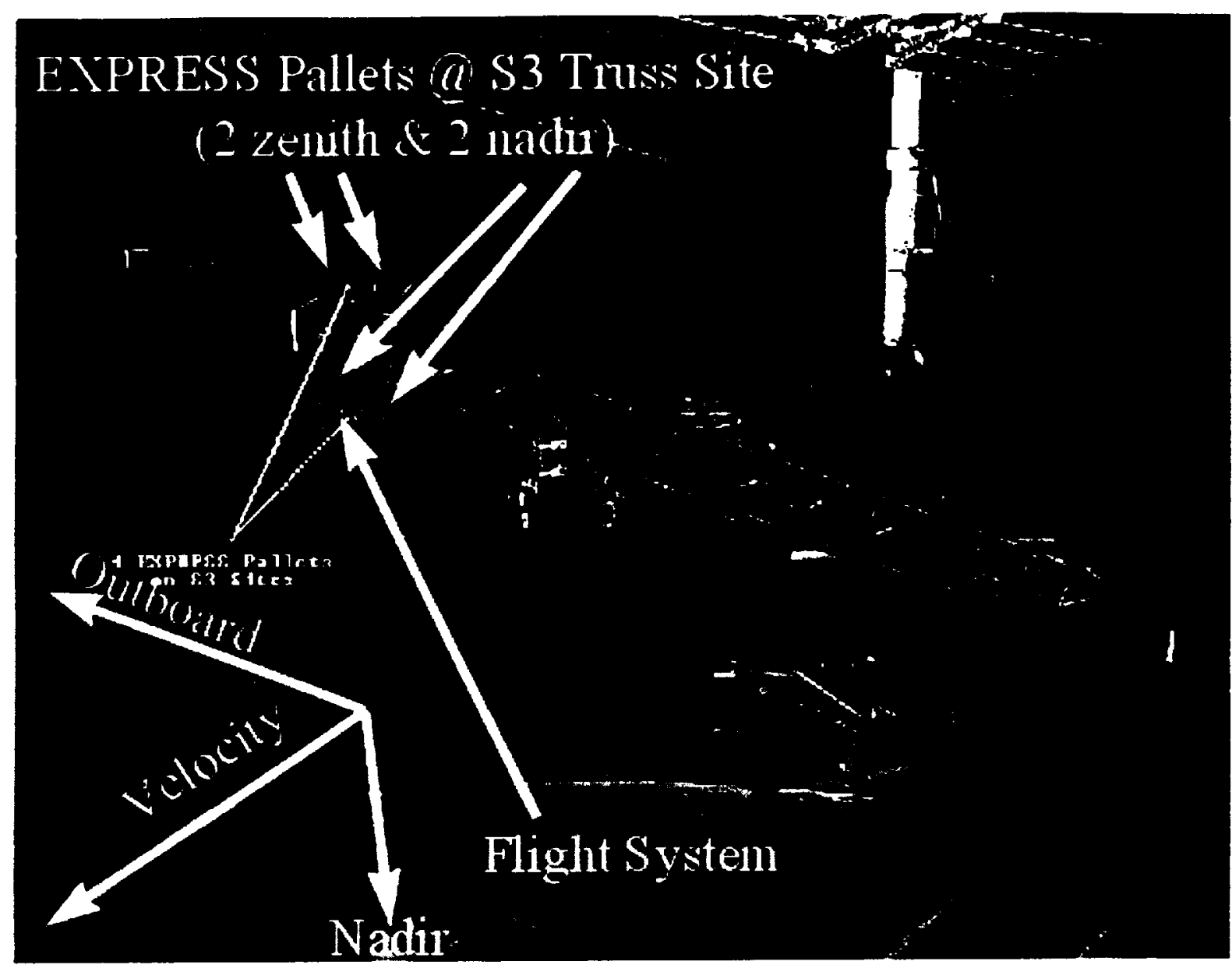

Figure 2 A conception of the International Space Station at assembly complete. The Flight System attaches to the nadir-facing outhoard EXPRESS Pallet Adapter at the port forward position. This location permits the Flight System to have an unobstructed line-of-sight to the rising of the Ground System for particular passes.

\section{FLIGHT SYSTEMI}

The Flight System of the OCDHRLF is manifested to be deployed 11 the ISS aboard Space Transportation System Utilization Flight 3 (STS-LF3), tentatively sehedule for Octoher 20r). The Flight System will be attached to a nadir outhoard EXpedite the PRocessing of Experiment w the Spate Station (FXPRESS) Pallet

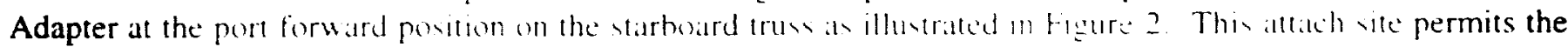

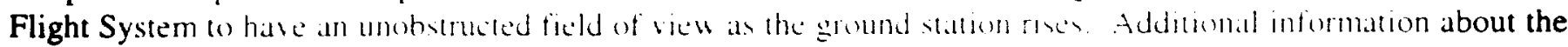
EXPRESS Pallet Adapter. which is the electrical. electronic. and phe sical connection hetucen the Flight System and the ISS, is contianed in refereme?

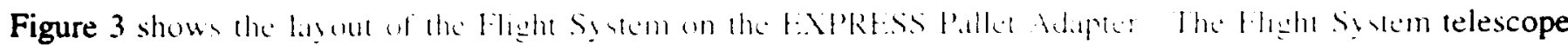

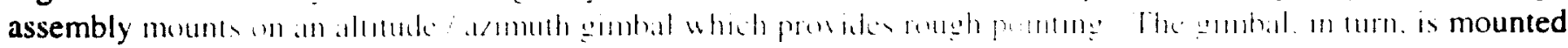

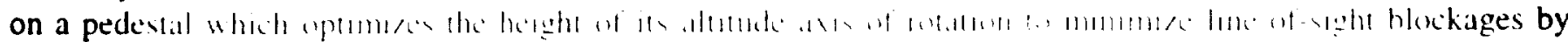

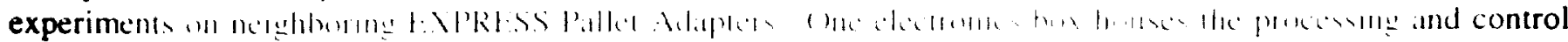

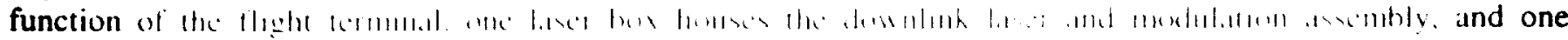

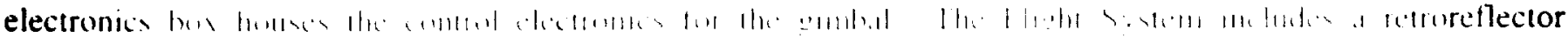




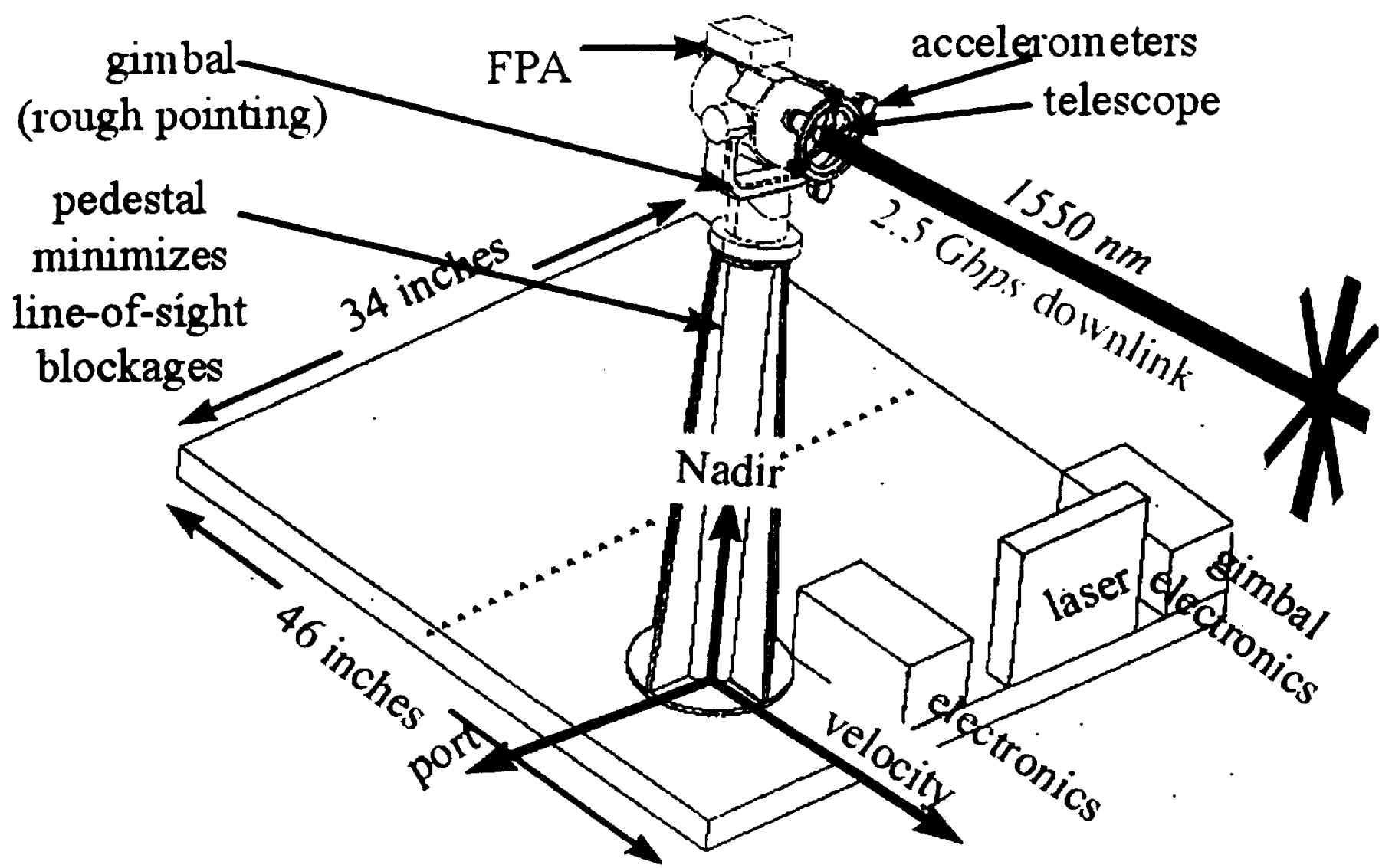

Figure 3. The Flight System integrated to an EXPRESS Pallet Adapter. The Flight System telescope assembly mounts on an altitude /azimuth gimbal which in turn mounts to a pedestal whose height minimizes line-of-sight blockages by neighboring payloads.

mounted on the floor of the EXPRESS Pallet Adapter which is designed to return the pulses at $1064 \mathrm{~nm}$ used by the ground station to verify that its telescope is correctly pointed at the Flight System. Three accelerometers mounted on the telescope assembly monitor telescope pointing changes induced by platform vibration.

The design of the Flight System retains the heritage architecture of the OCD laboratory-model form, fit, and function optical transmitter: fine tracking \& point-ahead using one focal-plane-array \& one fine steering mifror, and the downlink beam fiber-coupled to the telescope assembly. Since the design of the OCD has been discussed extensively in reference 5, it suffices to contrast the design of the Flight System with the design of the OCD. One key contrast is the operating wavelengths. The downlink beam of the Flight System is at the 'eye-safe' wavelength of $1550 \mathrm{~nm}$ and the beacon beam is at $980 \mathrm{~nm}$. The $980-\mathrm{nm}$ beacon wavelength choice allows the Ground System to utilize high-power commercially-produced lasers. Also, its long wavelength is favorable to scintillation effects yet remains visible to the FPA. The choice of $1550 \mathrm{~nm}$ for the downlink wavelength allows the Flight System to leverage the heavy investment that the fibercomm industry has made in the 1550-nm fiber laser system and at the same time makes the downlink beam eyesafe even directly in front of the Flight System aperture. The fibercomm industry investment in 1550-nm also allows the Ground System to minimize cost by utilizing a fibercomm COTS receiver package. Although the 1550 -nm downlink beam is not visible to the Flight System Focal Plane Array (FPA), the laser assembly includes a $980-\mathrm{nm}$ boresight reference beam co-propagating with the 1550-nm downlink. The FPA uses this boresight reference beam to perform tracking of the downlinked direction. This technique is further described in reference 7. 


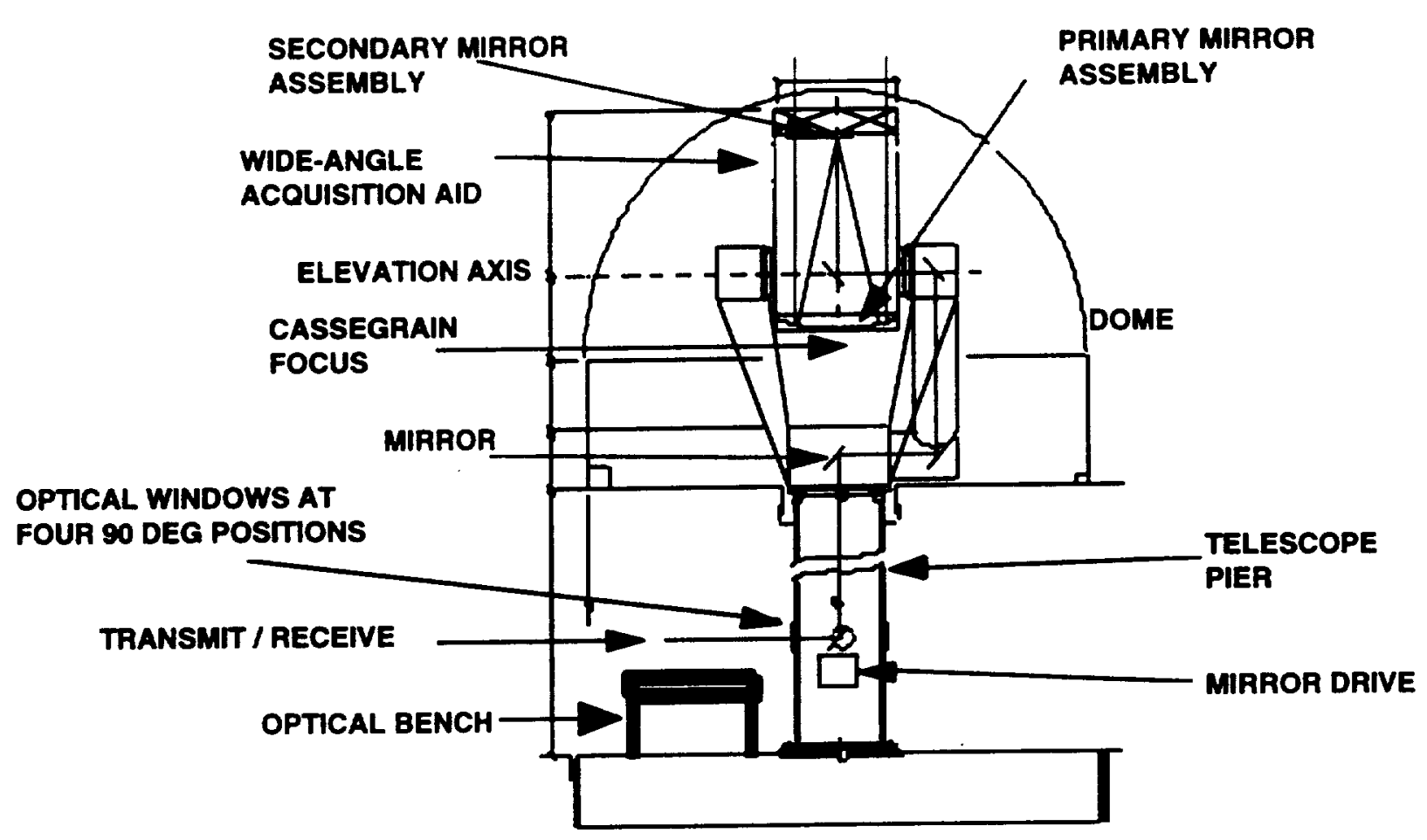

Figure 4. An illustration of the Optical Communication Telescope Laboratory to which the Ground System integrates.

Another key contrast between the design of the OCD and the preliminary design of the Flight System is the addition of a beacon monitor channel to the optical system. This channel splits off a portion of the beacon beam entering the telescope optical assembly and sends it to a detector which measures and digitizes the timedependent intensity changes cause by atmospheric fluctuations. Analysis of these scintillation measurements will enable the accuracy of atmospheric scintillation models to be tested and quantify the effectiveness of beaconbeam scintillation-mitigation techniques.

The electronics interface is another key change. The Flight System electronics interface has been expanded to include, in addition to the $1553 \mathrm{~B}$ command and control data which receives ISS position, attitude, roll rate, and time, as well as commands from the Ground System, an Ethernet connection for relaying high-rate enginetring data to the Ground System. This Ethernet connection enables measurements from the accelerometer array and scintillation monitor to be sent to the Ground System over the ExPA Ethemet link in near-real-time for recovery and analysis.

\section{GROUND SYSTEM}

The Ground System design draws heritage from the Ground-to-Orbit Lasercomm Demonstration ${ }^{8}$ and integrates to the OCTL, shown in Figure 4. The Ground System has three primary functions: maintain an illumination on the Flight System with the beacon beam, recover the downlink beam, and facilitate operations and planning.

The Ground System illuminates the Flight System with a beacon beam consisting of eight 1.25-W mutuallyincoherent $980-\mathrm{nm}$ laser beams broadcast from spatially separated locations on the primary mirror. The combination of these eight beams as they spatially overlap at the Flight System produces an irradiance whose scintillation is substantially reduced over the scintillation that an individual beam would posses. This spatial and 


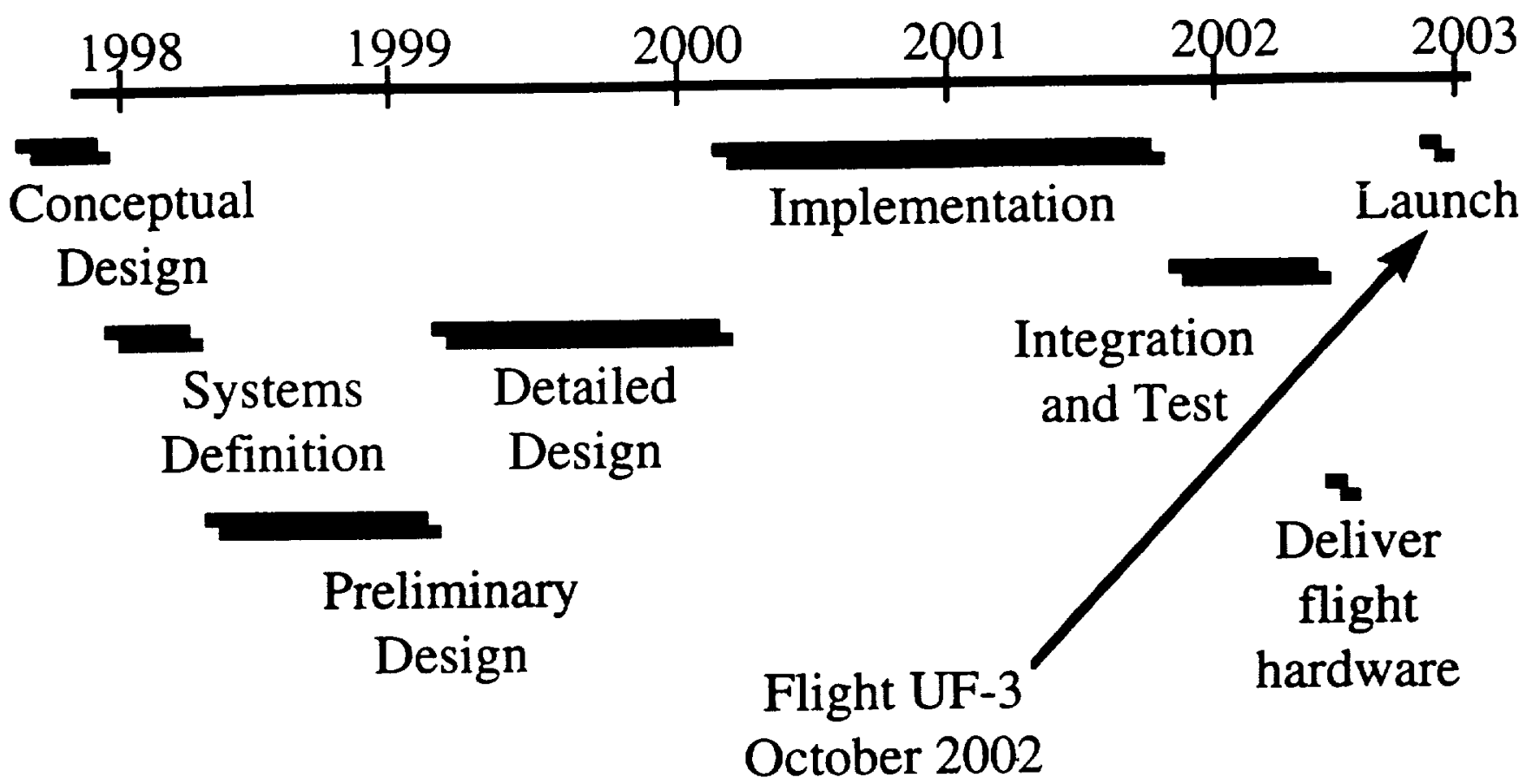

Figure 7. Schedule for the design and development of the flight terminal.

coherent separation technique, called beacon-beam averaging, was used successfully during the GOLD experiment to improve the signal quality received at the ETS-VI satellite. Each beacon laser has a divergence of $200 \mu \mathrm{rad}$ which is a compromise between the insensitivity to mispoint obtained with a broad beam and the beacon power required in a broad beam to maintain the required irradiance on the Flight System.

To ensure initial acquisition of the Flight System in the wide position uncertainty space defined by the 3000-ft 3sigma position knowledge accuracy of the ISS, the Ground System is capable of transmitting a 1-mrad-divergence beacon boresight beam consisting of pulses from a YAG laser. Observing the arrival of retroreflected pulses assures that the telescope is correctly pointed at the Flight System.

To receive the downlink communication beam, the Ground System receive channel focuses the downlink beam onto a COTS receiver unit appropriate to the data rates which the Flight System transmits. The receiver is followed by a bit-error-rate tester (BERT) which measures link performance. A scintillation monitor attached to the detector measures the downlink scintillation statistics for later comparison to theory. Data from these monitors will be correlated with atmospheric transmission measurements made by the autonomous visibility monitoring (AVM) ${ }^{9}$ station located near OCTL, further increasing our understanding of the systems performance.

Operations which involve the Flight System pass through the United States Ground Segment of the International Space Station Payload Operations and Integration Center (POIC) to be located at Marshall Space Flight Center. An Internet connection allows commands for the Flight System to be sent from OCTL through POIC to the Flight System. High-rate engineering data flowing down from the ISS Ethernet link is processed through POIC and provided in near-real-time to the OCTL.

\section{PLAN AND SC̈HEDULE}

The schedule for the development of the flight terminal is shown in Figure 5. The conceptual design, systems definition. and preliminary design have been completed. The detailed design phase culminates in a Critical 
Design Review (CDR) which will be followed by hardware fabrication and subsystem integration, leading to the integration and test of the Flight Terminal in early 2002. The complete flight terminal will be delivered to Kennedy Space Center six months before the October 2002 launch.

\section{SUMMARY}

In summary, the preliminary design of the Flight System of the Optical Communication Demonstration and HighRate Link Facility retains the heritage architecture of the Optical Communication Demonstrator while changing the downlink wavelength to $1550-\mathrm{nm}$, increasing the data rate to $2.488 \mathrm{Gbps}$, adding a beacon scintillation monitor and an accelerometer array, and changing the beacon-tracking wavelength to $980 \mathrm{~nm}$. The preliminary design of the Ground System is matched to the requirements of the Flight System and uses multiple beacon beams to reduce scintillation. The Flight System of the OCDHRLF is scheduled to be deployed to the ISS in October 2002.

\section{ACKNOWLEDGMENT:}

The research described in this paper was carried out at the Jet Propulsion Laboratory, California Institute of Technology, under contract with the National Aeronautics and Space Administration.

\section{REFERENCES}

${ }^{1}$ H. Hemmati, K. Wilson, M. Sue, D. Rascoe, F. Lansing. M. Wilhem, L. Harcke, and C. Chen, "Comparative Study of Optical and RF Communication Systems for a Mars Mission," SPIE Proceedings, Vol. 2699, pp. 146-164 (1996).

2 J. V. Sandusky and J. R. Lesh, "Planning for a Long-Term Optical Demonstration from the International Space Station," SPIE Proceedings, Vol. 3266, pp. 128-134 (1998).

${ }^{3}$ K. E. Wilson and J. V. Sandusky, "Development of a 1 -m-class telescope at TMF to support optical communications demonstrations," SPIE Proceedings Vol. 3266, pp. 146-152 (1998).

${ }^{4}$ U.S. Patent 5,517,016 "Lasercomm System Architecture with Reduced Complexity," May 14, 1996.

${ }^{5}$ N. A. Page, "Design of optical communications demonstrator instrument optical system," in Free Space Laser Communications Technology VI. OE-LASE'94, Jan 1994.

"Chen, C-C. and J. R. Lesh (1994) "Overview of the Optical Communications Demonstrator," in Proc. of SPIE OE-LASE 94, January 1994, Paper No. 2123-09.

' Gerardo G. Ortiz, Muthu Jeganathan, John V. Sandusky, and Hamid Hemmati, "Design of a 2.5 Gbps Optical Transmitter for the International Space Station", Proceedings of the SPIE, Vol. 3615 (1999).

James, J., Jeganathan, M., Toyoshima, M., Wilson, K., and Xu, G., "Data Analysis Results from the GOLD Experiments," SPIE Proceedings Vol. 2990 pp. 70 - 81 (1997).

${ }^{9}$ M. Jeganathan and D. Erickson, "Atmospheric Visibility Monitoring (AVM) Program," SPIE Proceedings Vol. 2990 (1997). 